Article

\title{
Co-Optimized Analysis and Design of Electric and Natural Gas Infrastructures
}

\author{
Santiago Lemos-Cano ${ }^{1, *}$ and James McCalley ${ }^{2, *}$ \\ 1 Empresa de Energía del Pacífico S.A. E.S.P, Medellín 050021, Colombia \\ 2 Department of Electrical and Computer Engineering, Iowa State University, Ames, IA 50011, USA \\ * Correspondence: slemoscano@gmail.com (S.L.-C.); jdm@iastate.edu (J.M.); Tel.: +57-4-326-6600 (S.L.-C.)
}

Received: 22 March 2019; Accepted: 8 May 2019; Published: 26 May 2019

\begin{abstract}
This paper proposes and implements a long-term deterministic capacity expansion model for the co-optimization of electric and natural gas infrastructures. It determines the required investments in generation units, transmission lines and pipelines for meeting future demands, while representing electricity and natural gas flows using DC Power Flow and Weymouth equations, respectively. A Mixed Integer Nonlinear Programming (MINLP) problem is developed, from which a linearized version is derived. A 26 node integrated gas-electric system for the Eastern Region of the United States is used to demonstrate the model's capabilities. Results show that the model provides an accurate operational representation of the integrated system, and, therefore, enhances the expansion planning process.
\end{abstract}

Keywords: co-optimization; long-term planning; capacity expansion; electric transmission; natural gas pipeline; disjunctive; power flow; Weymouth equations

\section{Introduction}

For many decades, natural gas has had an important role in the global energy landscape. It has traditionally been used within industrial processes and as a heating alternative for residential and commercial sectors. However, over the years, its participation as a fuel for generating electricity has increased considerably. There are multiple reasons associated with this phenomenon among which are: (i) the shale gas "boom", i.e., the significant increase of gas production due to technological developments in the extraction techniques of non-conventional resources; (ii) a reduction in the market prices as the result of the growth in the supply; (iii) the environmental benefits that combined cycle units have demonstrated when compared against other fossil-fired units; and (iv) the operational support that gas-fired units can provide for renewable integration.

Thus, it is clear that the electric sector has become an important player within the natural gas industry, which results in strong interactions between them, not only physically but also economically [1-4]. Ref. [5] presents a complete summary of different models reported in the literature, categorized by the type of analysis performed for integrated electricity and natural gas systems.

From an operational perspective, multiple research projects have been undertaken recently to analyze these interactions. A set of different methodologies and optimization problems have been proposed to quantify the flexibility that natural gas networks can provide to a power system, or to quantify the adequacy levels needed from gas systems to guarantee the reliability of a power system, under future generation scenarios considering intermittent renewable energy sources [6-9]. Coordinated scheduling strategies of electrical and natural gas resources in a day-ahead dispatch, including gas compressor operations, has also been addressed in the literature to analyze the economic and operational benefits of an integrated operation [10,11]. 
From a long-term planning perspective, multi-sector optimization models based on network flows were developed as an initial attempt to model these interactions by determining the combined infrastructure investment requirements [4,12-14]. However, these models cannot guarantee an accurate representation of electricity and natural gas flows across the transmission systems because they omit modeling of important physical laws. Ref. [15] derives a Mixed Integer Linear Programming (MILP) representation for the capacity expansion problem while considering the DC power flow equations for the transmission network to overcome this limitation. In addition, Mixed Integer Nonlinear Programming (MINLP) formulations have been derived in [16-20] for an integrated gas-electric representation.

This paper presents a disjunctive model using an MILP formulation that considers DC power flow and Weymouth equations in deterministic capacity expansion problems for integrated gas-electric systems. Initially, a disjunctive MINLP model is presented, which serves as the starting point for deriving a linearized representation using piecewise linear approximations. The capabilities of the proposed formulation are tested using a 26 node integrated gas-electric system simulated over 20 years.

The paper is structured as follows. Section 3 reviews the co-optimization expansion planning problem and presents a MINLP formulation for an integrated gas-electric system. Section 4 develops an initial MILP model using piecewise linear functions, while Section 5 presents an alternative, less computationally intensive formulation. Conclusions are shown in Section 6, after which the nomenclature used in the paper is provided.

\section{Co-Optimized Expansion Planning Problem}

A co-optimized expansion planning formulation provides simultaneous identification of two or more classes of related infrastructure decisions within a single optimization [21]. In this paper, we study the co-optimization expansion planning problem for an integrated gas-electric system. Hence, the deterministic model to be developed will aim to find the type, capacity, location, and timing of the required investments in generation units, transmission lines and pipelines that satisfy the future demands for both systems, while considering operational, security and reliability criteria.

Different representations for the electricity transmission network can be found in the literature [22], ranging from simple transportation models using network flows to more detailed formulations considering electric laws. Under steady state conditions, AC power flow equations provide an accurate representation of the electricity flows across the transmission network in planning studies.

However, this entails the need for considering a set of nonlinear/nonconvex equations in the optimization problem, with a considerable increase in their complexity. On the other hand, it is possible to use a transportation model at the expense, however, of violating Kirchhoff's Voltage Law, possibly causing an overestimation of electric transmission capabilities under some scenarios.

Gas transmission systems can also be modeled using different representations according to the assumptions considered. The simplest approach is to represent the pipeline network using a transportation model, which, as with the electric network, could overestimate gas transmission capabilities under some scenarios. Improvements can be obtained by considering gas flow steady state equations in pipelines, such as Weymouth equations, a set of nonlinear equations commonly used in the design of gas transmission networks.

A disjunctive MINLP formulation of the co-optimization expansion planning problem for an integrated gas-electric system is presented below, where electricity and gas flows are represented using DC Power Flow equations and Weymouth equations, respectively. Equation (1) presents the objective function, which expresses the net present value of the sum of operational costs (electricity generation and transmission as well as natural gas production and transmission), investment costs (for new generation units, transmission lines and pipelines), penalties (carbon emissions and unserved demand) and salvage values (for mitigating end horizon effects in long term planning simulations) of the integrated electric-gas system. Equations (2)-(29) constitute the mathematical formulation for the electric and gas systems. Equation (17) plays a significant role in that this constraint links both 
systems, by modeling the amount of gas required by the combustion turbine units and the combined cycle plants to generate electricity:

$$
\begin{aligned}
& \min \zeta=\sum_{\bar{g}, t} \xi^{t}\left(c_{\bar{g} t}^{f x, g} C_{\bar{\delta}}^{m a x} C_{\bar{g} t}+\sum_{m, s} c_{\bar{g} t}^{o p, g} P_{\bar{g} t m s}^{g} h_{s}\right)+\sum_{\bar{g}, t, m, s, f} \xi^{t} c_{t}^{c c} v_{k, f} \gamma_{\bar{g}} P_{\bar{g} t m s}^{g} h_{s} \\
& +\sum_{\bar{g}, t, m, s, f} \xi^{t} c_{\bar{g} f t m}^{o p, f \neq \text { gas }} \gamma_{\bar{g}} P_{\bar{g} t m s}^{g} h_{s}+\sum_{j, k, t, m, s} \xi^{t} c_{j t m}^{o p, p} \eta G_{j k t m s}^{p} h_{s}+\sum_{j, t, m, s} \xi^{t} c_{j t m}^{d, g a s} G_{j t m s}^{d} h_{s}
\end{aligned}
$$

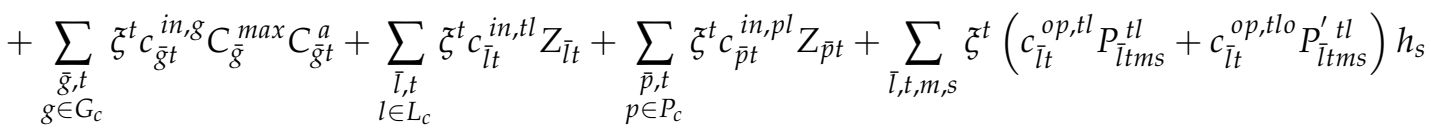

$$
\begin{aligned}
& +\sum_{\bar{p}, t, m, s} \xi^{t}\left(c_{\bar{p} t}^{o p, p l} G_{\bar{p} t m s}^{p l}+c_{\bar{p} t}^{o p, p l o} G_{\bar{p} t m s}^{\prime p l}\right) h_{s}+\sum_{j, t, m, s} \xi^{t} \tau P_{j t m s}^{l s} h_{s}+\sum_{\substack{\bar{d}, t, m, s \\
d \in D_{c}}} \xi^{t} c_{\bar{d} t}^{o p, c r}\left(\pi_{j^{\prime} t m s}-\pi_{j t m s}\right) h_{s} \\
& +\sum_{\substack{\bar{d}, t, m, s \\
d \in D_{r}}} \xi^{t} c_{\bar{d} t}^{o p, c r}\left(\pi_{j t m s}-\pi_{j^{\prime} t m s}\right) h_{s}-\sum_{\bar{g}, t} \xi^{l} c_{\bar{g} t}^{i n, g}\left(1-(\iota-(t-1)) / \delta_{k}^{g}\right) C_{\bar{g}}^{\max } C_{\bar{g} t}^{a} \\
& -\sum_{\bar{l}, t} \xi^{l} c_{\bar{l} t}^{i n, t l}\left(1-(\iota-(t-1)) / \delta^{t l}\right) Z_{\bar{l} t}-\sum_{\bar{p}, t} \xi^{l l} c_{\bar{p} t}^{i n, p l}\left(1-(\iota-(t-1)) / \delta^{p l}\right) Z_{\bar{p} t},
\end{aligned}
$$

subject to

$C_{\bar{g} t}=C_{\bar{g}}^{e}+\sum_{i=1}^{t}\left(C_{\bar{g} i}^{a}-C_{\bar{g} i}^{r}\right), \forall \bar{g}, t$,

$C_{\bar{g} t}^{a} \leq C_{\bar{g} t}^{a, m a x}, \forall \bar{g}, g \in g_{c}, t$,

$P_{\bar{g} t m s}^{g} \leq C C_{\bar{g} t m s} C_{\bar{g}}^{\max } C_{\bar{g} t}, \forall \bar{g}, t, m, s$,

$\sum_{m, S}\left(P_{\bar{g} t m s}^{g} h_{S}\right) \leq C F_{\bar{g} t} C_{\bar{g}}^{\max } C_{\bar{g} t} \sum_{m, S} h_{S}, \forall \bar{g}, t$,

$\sum_{\bar{g}} F C_{\bar{g} t} C_{\bar{g} t} \geq\left(1+r_{j}\right) P_{j t}^{d, p e a k}, \forall j \in J_{a}, t$

$\sum_{\bar{l}: B_{l}=j}-\left(P_{\bar{l} t m s}^{t l}-P_{\bar{l} t m s}^{t}\right)+\sum_{\bar{l}: E_{l}=j}\left(P_{\bar{l} t m s}^{t l}-P_{\bar{l} t m s}^{\prime} t l\right)=P_{j t m s}^{d}-P_{j t m s}^{l s}-\sum_{\bar{s}} P_{\bar{g} t m s}^{g}, \forall j \in J_{a}, t, m, s$,

$\theta_{B_{l} t m s}-\theta_{E_{l} t m s}=X_{\bar{l}}\left(\left(P_{\bar{l} t m s}^{t l}-P_{\bar{l} t m s}^{\prime t l}\right) / S^{b a s e}\right), \forall \bar{l} \in L_{e}, t, m, s$,

$-\left(1-S_{\bar{l} t}\right) M^{t l} \leq \theta_{B_{l} t m s}-\theta_{E_{l} t m s}-X_{\bar{l}}\left(\left(P_{\bar{l} t m s}^{t l}-P_{\bar{l} t m s}^{\prime t l}\right) / S^{\text {base }}\right) \leq\left(1-S_{\bar{l} t}\right) M^{t l}, \forall \bar{l} \in L_{c}, t, m, s$,

$S_{\bar{l} t}=\sum_{i=1}^{t} Z_{\bar{l} i}, \forall \bar{l} \in L_{c}, t$,

$P_{\bar{l} t m s}^{t l, m i n} \leq-P_{\bar{l} t m s}^{\prime} t l, \forall \bar{l} \in L_{e}, t, m, s$,

$P_{\bar{l} t m s}^{t l} \leq P_{\bar{l} t m s}^{t l, m a x}, \forall \bar{l} \in L_{e}, t, m, s$,

$S_{\bar{l} t} P_{\bar{l} t m s}^{t l, m i n} \leq-P_{\bar{l} t m s^{\prime}}^{\prime} t l, \forall \bar{l} \in L_{\mathcal{C}}, t, m, s$,

$P_{\bar{l} t m s}^{t l} \leq S_{\bar{l} t} P_{\bar{l} t m s}^{t l, m a x}, \forall \bar{l} \in L_{c}, t, m, s$,

$G_{j t m s}^{p, t} \leq G_{j t m s}^{p, \max }, \forall j \in J_{a}, t, m, s$, 
$\sum_{j \in J_{a}} G_{j k t m s}^{p}=\sum_{\bar{s}}\left(\frac{\gamma_{\bar{g}}}{\eta}\right) P_{\bar{g} t m s}^{g}, \forall k \in K_{g}, t, m, s$,

$\sum_{\bar{d}: B_{d}=j}-\left(G_{\bar{d} t m s}^{c r}-G_{\bar{d} t m s}^{\prime c r}\right)+\sum_{\bar{d}: E_{d}=j}\left(G_{\bar{d} t m s}^{c r}-G_{\bar{d} t m s}^{\prime c r}\right)=G_{j t m s}^{d}+\sum_{k \in K_{g}} G_{j k t m s}^{p}-G_{j t m s}^{p, t}, \forall j \in J_{a}, t, m, s$,

$\sum_{\bar{d}: B_{d}=j}-\left(G_{\bar{d} t m s}^{c r}-G_{\bar{d} t m s}^{\prime c r}\right)+\sum_{\bar{d}: E_{d}=j}\left(G_{\bar{d} t m s}^{c r}-G_{\bar{d} t m s}^{\prime c r}\right)-\sum_{\bar{p}: B_{p}=j}\left(G_{\bar{p} t m s}^{p l}-G_{\bar{p} t m s}^{\prime p l}\right)$

$+\sum_{\bar{p}: E_{p}=j}\left(G_{\bar{p} t m s}^{p l}-G_{\bar{p} t m s}^{\prime p l}\right)=0, \forall j \in J_{p}, t, m, s$,

$\pi_{B_{p} t m s}-\pi_{E_{p} t m s}-Y_{\bar{p}} G_{\bar{p} t m s}^{p l}\left|G_{\bar{p} t m s}^{p l}\right|=0, \forall \bar{p} \in P_{e}, t, m, s$,

$-\left(1-S_{\bar{p} t}\right) M^{p l} \leq \pi_{B_{p} t m s}-\pi_{E_{p} t m s}-Y_{\bar{p}} G_{\bar{p} t m s}^{p l}\left|G_{\bar{p} t m s}^{p l}\right| \leq\left(1-S_{\bar{p} t}\right) M^{p l}, \forall \bar{p} \in P_{c}, t, m, s$,

$S_{\bar{p} t}=\sum_{i=1}^{t} Z_{\bar{p} i}, \forall \bar{p} \in P_{c}, t$

$G_{\bar{p} t m s}^{p l, m i n} \leq-G_{\bar{p} t m s}^{\prime p l}, \forall \bar{p} \in P_{e}, t, m, s$,

$G_{\bar{p} t m s}^{p l} \leq G_{\bar{p} t m s}^{p l, m a x}, \forall \bar{p} \in P_{e}, t, m, s$,

$S_{\bar{p} t} G_{\bar{p} t m s}^{p l, m i n} \leq-G_{\bar{p} t m s}^{\prime p l}, \forall \bar{p} \in P_{c}, t, m, s$,

$G_{\bar{p} t m s}^{p l} \leq S_{\bar{p} t} G_{\bar{p} t m s}^{p l, m a x}, \forall \bar{p} \in P_{c}, t, m, s$,

$\pi_{j t m s} \leq \pi_{j^{\prime} t m s}, \forall \bar{d} \in D_{c}, t, m, s$,

$\pi_{j^{\prime} t m s} \leq W_{\bar{d}} \pi_{j t m s}, \forall \bar{d} \in D_{c}, t, m, s$,

$\pi_{j^{\prime} t m s} \leq \pi_{j t m s}, \forall \bar{d} \in D_{r}, t, m, s$,

$\pi_{j t m s} \leq W_{\bar{d}} \pi_{j^{\prime} t m s}, \forall \bar{d} \in D_{r}, t, m, s$,

$-1.57 \leq \theta_{j t m s} \leq 1.57, \forall j \in J_{a}, t, m, s$,

$\pi_{j t m s}^{\min } \leq \pi_{j t m s} \leq \pi_{j t m s}^{\max }, \forall j, t, m, s$,

$C_{\bar{g} t}, P_{\bar{g} t m s}^{g}, P_{j t m s}^{l s}, P_{\bar{l} t m s^{\prime}}^{t l} P_{\bar{l} t m s}^{\prime t l} \geq 0$,

$G_{j t m s^{\prime}}^{p, t} G_{j k t m s^{\prime}}^{p} G_{\bar{p} t m s^{\prime}}^{p l} G_{\bar{p} t m s^{\prime}}^{\prime p l}, G_{\bar{d} t m s^{\prime}}^{c r} G_{\bar{d} t m s}^{\prime c r} \geq 0$,

Integer variables: $C_{\bar{g} t}^{a}, C_{\bar{g} t}^{r}$,

Binary variables: $S_{\bar{l} t}, Z_{\bar{l} t}, S_{\bar{p} t}, Z_{\bar{p} t}$.

Integer variables are used for modeling generation additions and retirements; therefore, Equation (2) allows determination of the number of units available at every time step. Equation (3) imposes an upper bound for new generation investments. Equations (4) and (5) limit power and 
electricity outputs from the units using capacity credit and capacity factor parameters, respectively. In particular, Equation (4) accounts for the tendency of each generator to operate within certain ranges during certain time periods, while Equation (5) accounts for the tendency of each generator to produce over a year a certain fraction of the energy it would produce if it is continuously operated at its capacity during that time frame. Additionally, Equation (6) enforces a reliability criterion by requiring a minimum amount of capacity reserves in each region using a peak power demand value.

Equations (7)-(14) represent the electric system transmission network using a disjunctive approach as proposed in [15]. Equation (7) enforces power balance in each area. Equations (8) and (9) represent the DC power flow equations for the existing and candidate transmission lines, respectively. Equation (10) tracks the investments in transmission lines in every time step. Equations (11)-(14) constrain the amount of power that can be transferred across each transmission line in the electric system. A sufficiently large parameter $M^{t l}$ is required in Equation (9). Although not essential, computational benefit can result from choosing this parameter according to the rationale given in [23] as explained in [24].

Natural gas production is represented using Equations (15) and (16). Equation (15) imposes upper bound limits for gas production levels while Equation (16) enforces a global balance between the amount of gas produced with generation purposes and the gas consumed by each type of generation technology.

Equations (17)-(29) define a disjunctive model for the pipeline network of the natural gas system. In this formulation, every natural gas transmission link includes one or more pipelines (considering existing and candidate) which are coupled with compression and reduction stations located at the start and end nodes, respectively, as illustrated in Figure 1. By using these new elements, pressure variables are adequately controlled across the pipeline network, increasing not only the system operational flexibility but also the accuracy of the investment decisions.

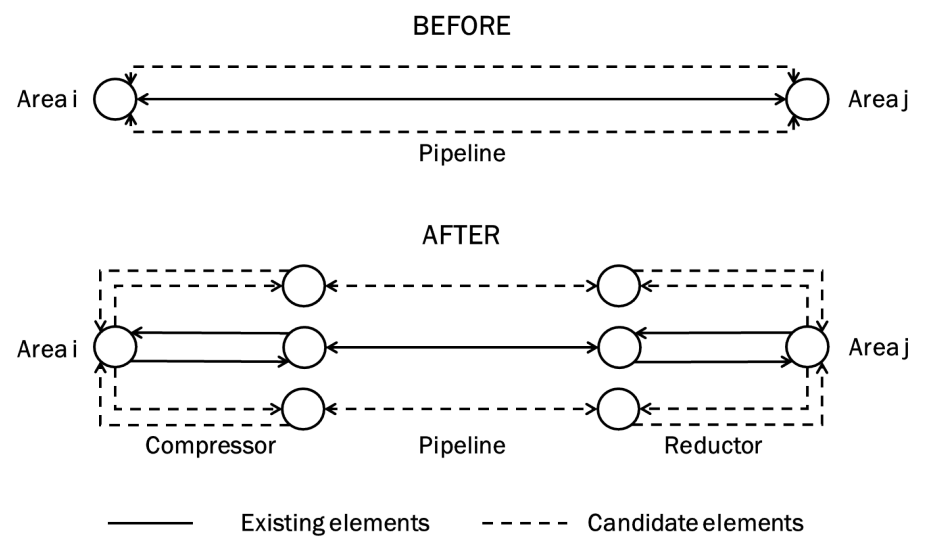

Figure 1. Natural gas transmission link representation.

Equations (17) and (18) enforce gas balances in area and pipeline nodes, respectively, while Equations (19) and (20) represent Weymouth equations for existing and candidate pipelines. Similar to the disjunctive model proposed for the electric transmission system, Equation (21) tracks the investments in pipelines, while Equations (22)-(25) limit the amount of gas that can be transferred using the pipeline network. Once again, a large parameter $M^{p l}$ must be used in Equation (20) to relax the pressure differences across the candidate pipelines when these have not been built yet.

Equations (26)-(29) describe the operation of the compression and reduction stations as a function of the pressure variables at the terminal points of the pipelines. For compression stations, the pressure at the receiving node has to be equal to or greater than the pressure at the sending node. In addition, the former cannot exceed the latter scaled by the compression ratio. For reduction stations, the pressure at the sending node has to be equal to or greater than the pressure at the receiving node, and the latter has to be at least equal to the former scaled by the inverse of the compression ratio. 
Finally, Equations (30)-(35) establish upper and lower limits for some of the decision variables, impose non-negativity requirements, and define the required binary and integer variables. The proposed model is a MINLP formulation. To facilitate its solution, we approximate it with an MILP formulation as described in the following sections.

\section{Co-Optimized Expansion Planning Problem Using Piecewise Linear Functions}

The MINLP problem described in the previous section is challenging to solve. We do so by replacing its nonlinear functions with piecewise linear ones that reasonably capture the nonlinearities associated with the original problem. This enables us to take advantage of the maturity of the algorithms developed to solve MILP problems. There are multiple approaches for linearizing bivariate nonlinear functions, one of which is described as follows. Consider a bivariate nonlinear function $h: \Omega \subseteq \Re^{2} \rightarrow \Re$ with $\Omega=\left\{(x, y) \backslash x_{a} \leq x \leq x_{b}, y_{a} \leq y \leq y_{b}\right\}$. Partitioning $\Omega$ as $i \in \Lambda_{x}=\left\{1, \ldots, k_{x}\right\}$, and $j \in \Lambda_{y}=\left\{1, \ldots, k_{y}\right\}$, it is possible to represent the value of any point $(x, y)$ enclosed inside the rectangle $\Delta_{i j}=\left\{(x, y) / x_{i} \leq x \leq x_{i+1}, y_{j} \leq y \leq y_{j+1}\right\}$ using a convex combination of the coordinates associated with its corners. Moreover, it is also possible to approximate $h$ using piecewise linear functions defined for each of multiple rectangles in the domain $\Omega$. Following this procedure, the surface defined by the equation $h(x, y)=0$ is approximated using a set of rectangular planes joined together by their corners.

In general, Equations (36)-(40) can be used to approximate constraints of the form $h(x, y)=0$ (where $h$ is a nonlinear function), in optimization problems using Special Ordered Set Type 2 (SOS2) variables [25]:

$x=\sum_{j \in \Lambda_{x}} \lambda_{i}^{x} x_{i}, y=\sum_{j \in \Lambda_{y}} \lambda_{j}^{y} y_{j}$

$h(x, y) \approx \sum_{i \in \Lambda_{x}} \sum_{j \in \Lambda_{y}} \lambda_{i j}^{h} h_{i j}$

$\sum_{i \in \Lambda_{x}} \lambda_{i}^{x}=1, \sum_{j \in \Lambda_{y}} \lambda_{j}^{y}=1$,

$\sum_{i \in \Lambda_{x}} \lambda_{i j}^{h}=\lambda_{i}^{y} \ldots \forall j \in \Lambda_{y}, \quad \sum_{j \in \Lambda_{y}} \lambda_{i j}^{h}=\lambda_{i}^{x} \ldots \forall i \in \Lambda_{x}$

$\lambda_{i}^{x}, \lambda_{j}^{y}, \lambda_{i j}^{h} \geq 0, \lambda_{i}^{x}, \lambda_{j}^{y}$ SOS2 variables.

We applied this approach to approximate the Weymouth equations, which are bivariate nonlinear functions relating gas flows to pressure values at the terminals of a pipeline. The MINLP problem presented in Section 2 can be reformulated as an MILP model by replacing Equations (19) and (20) with Equations (41)-(49):

$$
\begin{aligned}
& \pi_{j t m s}=\sum_{i} \lambda \pi_{j t m s i} \Pi_{j i}, \forall j, t, m, s, \\
& G_{\bar{p} t m s}^{p l}-G_{\bar{p} t m s}^{\prime p l}=\sum_{i} \sum_{i^{\prime}} \lambda g_{\bar{p} t m s i i^{\prime}}^{p l} g_{\bar{p} t m s i i^{\prime}}^{v a l}, \forall \bar{p}, t, m, s, \\
& \sum_{i} \lambda \pi_{j t m s i}=1, \forall j, t, m, s, \\
& \sum_{i^{\prime}} \lambda g_{\bar{p} t m s i i^{\prime}}^{p l}=\lambda \pi_{j t m s i}, \forall \bar{p} \in P_{e}, t, m, s, i, \\
& \sum_{i} \lambda g_{\bar{p} t m s i i^{\prime}}^{p l}=\lambda \pi_{j t m s i^{\prime}}, \forall \bar{p} \in P_{e}, t, m, s, i^{\prime},
\end{aligned}
$$




$$
\begin{aligned}
& -\left(1-S_{\bar{p} t}\right) M^{p l}+\lambda \pi_{j t m s i} \leq \sum_{i^{\prime}} \lambda g_{\bar{p} t m s i i^{\prime}}^{p l} \leq \lambda \pi_{j t m s i}+\left(1-S_{\bar{p} t}\right) M^{p l}, \forall \bar{p} \in P_{c}, t, m, s, i, \\
& -\left(1-S_{\bar{p} t}\right) M^{p l}+\lambda \pi_{j t m s i^{\prime}} \leq \sum_{i} \lambda g_{\bar{p} t m s i i^{\prime}}^{p l} \leq \lambda \pi_{j t m s i^{\prime}}+\left(1-S_{\bar{p} t}\right) M^{p l}, \forall \bar{p} \in P_{c}, t, m, s, i^{\prime}, \\
& \lambda \pi_{j t m s i}, \lambda g_{\bar{p} t m s i i^{\prime}}^{p l} \geq 0, \\
& \lambda \pi_{j t m s i} \text { SOS2 variables. }
\end{aligned}
$$

Equations (41)-(49) constitute a disjunctive MILP capacity expansion model, representing the natural gas flows using piecewise linear functions via a partition of the pressure variables in the pipeline network. Equations (41) and (42) correspond with Equations (36)-(37) which are linear combinations for calculating pressures and gas flows in the pipeline network, provided the unknown coefficients $\lambda \pi_{j t m s i}$ and $\lambda g_{\bar{p} t m s i i^{\prime}}^{p l}$. Equations (43)-(45) correspond with Equations (38) and (39) and establish a set of relations that parameters $\lambda \pi_{j t m s i}$ and $\lambda g_{\bar{p} t m s i i^{\prime}}^{p l}$ must satisfy in order to be able to adequately define the set of piecewise linear functions. Equations (46) and (47) enforce Weymouth equations for candidate pipelines. Recalling that $S_{\bar{p} t}$ is a binary variable used to track if candidate pipeline $\bar{p}$ has or has not been installed until period $t$, therefore, when $S_{\bar{p} t}=1$, Equations (46) and (47) reduce to Equations (44) and (45), and enforce the Weymouth equation for $\bar{p}$. However, if $S_{\bar{p} t}=0$, Equations (46) and (47) reduce to Equations (50) and (51), which in conjunction with Equations (24), (25) and (42) will set variables $\lambda g_{\bar{p} t m s i i^{\prime}}^{p l}$ to zero for $\bar{p}$, while $\lambda \pi_{j t m s i}$ and $\lambda \pi_{j t m s i^{\prime}}$ still can take nonzero values:

$$
\begin{aligned}
& -M^{p l}+\lambda \pi_{j t m s i} \leq \sum_{i^{\prime}} \lambda g_{d t m s i i^{\prime}}^{p l} \leq \lambda \pi_{j t m s i}+M^{p l}, \\
& -M^{p l}+\lambda \pi_{j t m s i^{\prime}} \leq \sum_{i} \lambda g_{d t m s i i^{\prime}}^{p l} \leq \lambda \pi_{j t m s i^{\prime}}+M^{p l} .
\end{aligned}
$$

Therefore, it is possible to approximate the MINLP problem presented in Section 2 with an MILP by using Equations (41)-(49) instead of Equations (19) and (20).

\section{Alternative Formulation Using Piecewise Linear Functions}

Although the MILP formulation presented in Section 3 is a novel approximation for solving the co-optimized expansion planning problem for an integrated electric-gas system, it is also a computationally intensive one (it requires an SOS2 variable for each $\lambda \pi_{j t m s i}$ coefficient), and therefore for large co-optimization problems, particularly ones with large number of candidate transmission lines (and therefore large number of integer variables), it requires significant computation just to find a feasible solution. We address this by developing a different MILP approximation.

It is possible to reduce the number of SOS2 variables required by representing the gas flow terms in the Weymouth equations directly as piecewise linear functions [26], as presented in Equations (52)-(54). This means that Equations (21)-(25) in conjunction with Equations (52)-(58) will constitute an alternative disjunctive formulation for a pipeline network; however, it is also a less accurate representation because of the linear approximation used in Equation (53):

$$
\begin{aligned}
& G_{\bar{p} t m s}^{p l}-G_{\bar{p} t m s}^{\prime p l}=\sum_{i} \lambda g_{\bar{p} t m s i}^{p l} g_{\bar{p} t m s i}^{v a l}, \forall \bar{p}, t, m, s, \\
& \Gamma_{\bar{p} t m s}^{p l}=\sum_{i} \lambda g_{\bar{p} t m s i}^{p l}\left(g_{\bar{p} t m s i}^{v a l}\right)^{2}, \forall \bar{p}, t, m, s, \\
& \sum_{i} \lambda g_{\bar{p} t m s i}^{p l}=1, \forall \bar{p}, t, m, s,
\end{aligned}
$$


$\pi_{B_{p} t m s}-\pi_{E_{p} t m s}=Y_{\bar{p}} \Gamma_{\bar{p} t m s}^{p l}, \forall \bar{p} \in P_{e}, t, m, s$,

$-\left(1-S_{\bar{p} t}\right) M^{p l} \leq \pi_{B_{p} t m s}-\pi_{E_{p} t m s}-Y_{\bar{p}} \Gamma_{\bar{p} t m s}^{p l} \leq\left(1-S_{\bar{p} t}\right) M^{p l}, \forall \bar{p} \in P_{c}, t, m, s$,

$\lambda g_{\bar{p} t m s i}^{p l} \geq 0, \forall \bar{p}, t, m, s$,

$\lambda g_{\bar{p} t m s i}^{p l} S O S 2$ variables.

\section{Results}

\subsection{Model Results}

The 26 node electric system described in [27] is modified and extended by creating a more disaggregated dataset for the generation plants, transmission lines and pipelines, using the additional information resources provided in [28] to represent an integrated gas-electric system for the region of the Eastern Interconnection in the United States, which in turn is used to demonstrate the capabilities of the proposed formulation in a 20-year simulation. The optimization problem is formulated using the software GAMS, and it is optimized using the solver CPLEX.

A high renewable scenario is created by imposing carbon emission costs to fossil fuel generation, restricting new investments in nuclear plants and allowing significant developments of wind farms. Final generation capacity values are summarized in Table 1, respectively, aggregated under six different technology groups (biomass, geothermal, landfill gas, solar, and oil/wood steam turbines are grouped under the label Others). Large investments in gas and wind technologies can be observed in the simulation results. New gas plants are built close to the new shale areas, or to areas connected to them using pipelines. Wind generation is developed strongly in the Midwest region, mainly due to the high capacity factor values of the technology in this area.

Table 1. Final generation capacity values (GW).

\begin{tabular}{lcccccc}
\hline \multirow{2}{*}{ Region } & \multicolumn{5}{c}{ Generation Technologies } \\
\cline { 2 - 7 } & Coal & Gas & Hydro & Nuclear & Others & Wind \\
\hline ENT & 0.7 & 24.6 & 1.9 & 5.3 & 0.3 & 0.1 \\
FRCC & 0.5 & 28.3 & 0.1 & 24.4 & 0.9 & 0.0 \\
HQ & 0.0 & 0.0 & 4.8 & 0.0 & 0.0 & 0.0 \\
IESO & 0.0 & 3.6 & 9.1 & 11.5 & 1.5 & 5.0 \\
MAPP_CA & 0.0 & 2.7 & 7.2 & 0.0 & 0.0 & 0.6 \\
MAPP_US & 0.0 & 2.1 & 2.2 & 0.5 & 0.2 & 9.9 \\
MISO_IN & 0.6 & 6.4 & 0.1 & 0.0 & 0.1 & 11.5 \\
MISO_MI & 0.0 & 24.9 & 2.0 & 1.9 & 0.5 & 16.0 \\
MISO_MO-IL & 1.8 & 6.9 & 1.1 & 2.2 & 0.5 & 28.3 \\
MISO_W & 0.0 & 16.6 & 0.5 & 2.3 & 0.5 & 136.2 \\
MISO_WUMS & 0.5 & 9.8 & 0.3 & 1.6 & 0.2 & 3.3 \\
NB & 0.0 & 0.0 & 0.0 & 0.0 & 0.0 & 0.0 \\
NE & 0.2 & 7.1 & 0.2 & 1.3 & 0.1 & 17.9 \\
NEISO & 0.0 & 10.5 & 6.3 & 4.6 & 1.6 & 4.9 \\
NonRTO_Midwest & 0.0 & 11.5 & 0.9 & 0.0 & 0.1 & 0.1 \\
NYISO_A-F & 0.0 & 1.8 & 5.8 & 3.2 & 0.6 & 6.7 \\
NYISO_G-I & 0.0 & 2.7 & 0.0 & 2.0 & 0.3 & 0.2 \\
NYISO_J-K & 0.0 & 14.7 & 0.0 & 0.0 & 0.6 & 0.0 \\
PJM_E & 0.0 & 19.7 & 0.7 & 8.5 & 0.7 & 8.6 \\
PJM_ROM & 0.5 & 7.9 & 3.6 & 5.0 & 0.7 & 18.1 \\
PJM_ROR & 2.2 & 72.1 & 5.4 & 20.0 & 1.5 & 19.1 \\
SOCO & 0.5 & 35.0 & 6.7 & 14.5 & 0.5 & 0.0 \\
SPP_N & 0.5 & 11.3 & 0.0 & 1.2 & 0.1 & 41.6 \\
SPP_S & 1.0 & 20.2 & 2.6 & 0.0 & 0.1 & 43.5 \\
TVA & 1.0 & 19.6 & 6.9 & 7.9 & 0.3 & 0.0 \\
VACAR & 1.3 & 23.9 & 4.8 & 13.8 & 0.4 & 30.0 \\
\hline
\end{tabular}


Initial transmission capacities and new investments in lines and pipelines are presented in Tables 2 and 3 (an NA label means that the transmission link is not represented either as an existing or as a candidate element in the model). Large investments in transmission lines to move the electricity produced by the new wind farms from the Midwest region to the main load centers located in the East Coast are reported in the results, as well as significant builds on new pipelines to move shale gas resources to regions with high gas generation capacities.

A high level and aggregated design for an integrated gas-electric transmission network according to the co-optimization results is depicted in Figure 2. Final generation capacities aggregated by super regions (as described in Table 4) for three of the six generation group technologies (coal, gas and wind) are also presented. The figure illustrates strong correspondence between the required investments in generation, transmission lines and pipelines. This design proposes a robust electric transmission grid configured as a ring between MISO and PJM to move the large amounts of electricity produced in the new wind farms. It also requires a robust pipeline network connecting the regions South, PJM and North to transport the shale gas in the south-north direction.

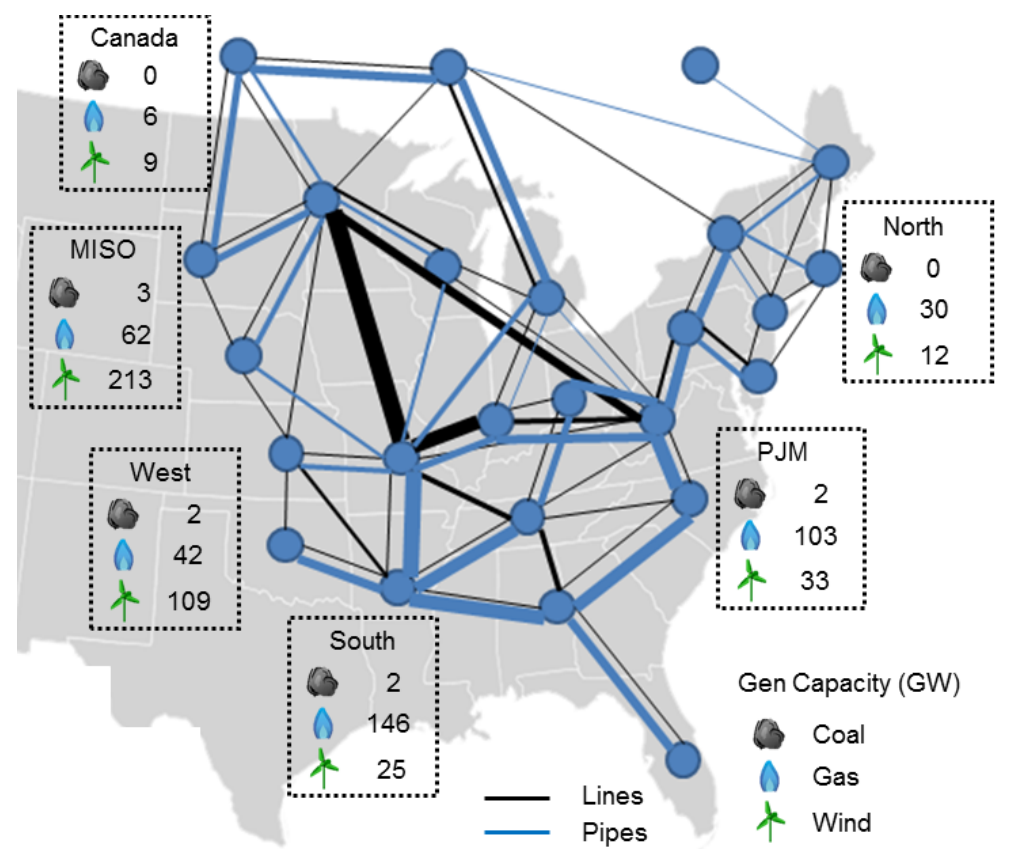

Figure 2. Integrated gas-electric network.

Table 2. Transportation initial capacity and investments.

\begin{tabular}{llccc}
\hline \multirow{2}{*}{ Link } & \multicolumn{2}{c}{ Lines (GW) } & \multicolumn{2}{c}{ Pipes (MMcf/h) } \\
\cline { 2 - 5 } & Initial & Invest. & Initial & Invest. \\
\hline ENT to MISO_MO-IL & 2.5 & 0.0 & 265.6 & 63.0 \\
ENT to SOCO & 2.4 & 0.0 & 312.5 & 0.0 \\
ENT to SPP_N & 7.3 & 0.0 & NA & NA \\
ENT to SPP_S & 4.3 & 2.0 & 208.3 & 0.0 \\
ENT to TVA & 3.0 & 0.0 & 265.6 & 0.0 \\
FRCC to SOCO & 3.7 & 0.0 & 215.0 & 0.0 \\
HQ to NEISO & NA & NA & 8.3 & 0.0 \\
IESO to MAPP_CA & 0.3 & 0.0 & 187.5 & 0.0 \\
IESO to MISO_MI & 3.1 & 4.0 & 125.0 & 63.0 \\
IESO to MISO_W & 0.2 & 0.0 & NA & NA \\
IESO to NEISO & NA & NA & 31.3 & 21.0 \\
IESO to NYISO_A-F & 2.2 & 0.0 & NA & NA \\
MAPP_CA to MAPP_US & 0.4 & 0.0 & 156.3 & 0.0 \\
\hline
\end{tabular}


Table 3. Transportation initial capacity and investments (continuation).

\begin{tabular}{llccc}
\hline & \multicolumn{3}{c}{ Lines (GW) } & Pipes (MMcf/h) \\
\cline { 2 - 5 } Link & Initial & Invest. & Initial & Invest. \\
\hline MAPP_CA to MISO_W & 2.0 & 0.0 & 93.8 & 0.0 \\
MAPP_US to MISO_W & 2.6 & 0.0 & 156.3 & 0.0 \\
MAPP_US to NE & 2.0 & 0.0 & NA & NA \\
MISO_IN to MISO_MI & 5.5 & 0.0 & 41.7 & 0.0 \\
MISO_IN to MISO_MO-IL & 5.0 & 24.0 & 156.3 & 0.0 \\
MISO_IN to NonRTO_Midwest & 4.8 & 0.0 & NA & NA \\
MISO_IN to PJM_ROR & 1.0 & 12.0 & 187.5 & 0.0 \\
MISO_MI to MISO_MO-IL & NA & NA & 104.2 & 0.0 \\
MISO_MI to MISO_WUMS & 0.3 & 0.0 & NA & NA \\
MISO_MI to PJM_ROR & 1.4 & 2.0 & 41.7 & 0.0 \\
MISO_MO-IL to MISO_W & 4.0 & 34.0 & NA & NA \\
MISO_MO-IL to MISO_WUMS & NA & NA & 62.5 & 0.0 \\
MISO_MO-IL to NE & NA & NA & 62.5 & 0.0 \\
MISO_MO-IL to PJM_ROR & 1.2 & 0.0 & NA & NA \\
MISO_MO-IL to SPP_N & 4.0 & 2.0 & 114.6 & 0.0 \\
MISO_MO-IL to TVA & 4.0 & 10.0 & NA & NA \\
MISO_W to MISO_WUMS & 1.7 & 6.0 & 93.8 & 0.0 \\
MISO_W to NE & 3.6 & 0.0 & 133.3 & 0.0 \\
MISO_W to PJM_ROR & 19.8 & 0.0 & NA & NA \\
MISO_W to SPP_N & 3.2 & 0.0 & NA & NA \\
MISO_WUMS to PJM_ROR & 1.6 & 0.0 & NA & NA \\
NE to SPP_N & 1.9 & 2.0 & NA & NA \\
NEISO to NYISO_A-F & 0.6 & 0.0 & 93.8 & 0.0 \\
NEISO to NYISO_G-I & 0.6 & 0.0 & NA & NA \\
NEISO to NYISO_J-K & 1.0 & 0.0 & NA & NA \\
NonRTO_Midwest to PJM_ROR & NA & NA & 156.3 & 42.0 \\
NonRTO_Midwest to TVA & 2.4 & 0.0 & 166.7 & 0.0 \\
NYISO_A-F to NYISO_G-I & 5.3 & 2.0 & 20.8 & 0.0 \\
NYISO_A-F to NYISO_J-K & NA & NA & 20.8 & 42.0 \\
NYISO_A-F to PJM_ROM & 2.0 & 0.0 & 187.5 & 0.0 \\
NYISO_G-I to NYISO_J-K & 6.1 & 2.0 & NA & NA \\
NYISO_G-I to PJM_E & 1.5 & 0.0 & NA & NA \\
NYISO_J-K to PJM_E & 0.3 & 0.0 & NA & NA \\
PJM_E to PJM_ROM & 8.0 & 0.0 & 125.0 & 0.0 \\
PJM_ROM to PJM_ROR & 8.0 & 0.0 & 250.0 & 0.0 \\
PJM_ROR to TVA & 2.5 & 0.0 & NA & NA \\
PJM_ROR to VACAR & 3.0 & 0.0 & 125.0 & 189.0 \\
SOCO to TVA & 3.2 & 10.0 & NA & NA \\
SOCO to VACAR & 3.0 & 0.0 & 156.0 & 126.0 \\
SPP_N to SPP_S & 4.0 & 2.0 & NA & NA \\
TVA to VACAR & 0.9 & 0.0 & NA & NA \\
\hline & & & & \\
\hline
\end{tabular}

Table 4. Super regions' description.

\begin{tabular}{ll}
\hline Super Regions & \multicolumn{1}{c}{ Areas } \\
\hline Canada & HQ, IESO, MAPP_CA \\
MISO & MISO (IN, MI, MO-IL, W, WUMS) \\
North & NEISO, NYISO (A-F, G-I, J-K) \\
PJM & PJM (E, ROM, ROR) \\
West & MAPP_US, NE, SPP (N, S) \\
South & ENT, FRCC, NonRTO Midwest, SOCO, TVA, VACAR \\
\hline
\end{tabular}

\subsection{Comparative Results}

Tables 5-7 compare the results from three different long-term capacity expansion models: a Pipes and Bubbles approach (also known as a transportation model) for an electric system (Model 1); a Pipes and Bubbles approach for an integrated electric-gas system (Model 2); and the disjunctive model for an integrated electric-gas system developed throughout this paper (Model 3). The comparison between 
the results obtained for models 1 and 2 explains the impact of considering the pipeline network in the co-optimization problem; similarly, the effect of modeling the steady state flow equations for electricity and natural gas is pointed out by comparing models 2 and 3 .

The inclusion of the pipeline network in Model 2 allows the decrease of the total cost of the co-optimization problem when compared with Model 1. This reduction is mainly due to a better utilization of the available gas resources between areas, and the decrease of the O\&M costs for the generation plants because of a more economical selection of the investments. This is not necessarily the best environmental choice. Notice that, under this investment plan, some wind farms are replaced by gas-fired units, which marginally increase the penalties associated with carbon dioxide emissions.

As expected, Model 3 has the highest total cost. From a mathematical point of view, this phenomenon is explained by the increased number of constraints. From an operational point of view, the addition of the steady state flow equations limits the amount of electricity and natural gas that can be sent through the transmission links, requiring more investments in generation and transmission, and increasing the O\&M costs. Therefore, in order to optimize system operation under the new constraints, some of the investments in new wind farms and gas-fired plants must be replaced by other generation technologies, and some new transmission lines are replaced by new pipelines.

Table 5. Cost comparison (dollars).

\begin{tabular}{|c|c|c|c|c|}
\hline \multicolumn{2}{|c|}{ Costs } & Model 1 & Model 2 & Model 3 \\
\hline \multirow{3}{*}{ O\&M } & Generation & $9.850 \times 10^{11}$ & $9.684 \times 10^{11}$ & $1.008 \times 10^{12}$ \\
\hline & Lines & $2.147 \times 10^{10}$ & $2.008 \times 10^{10}$ & $1.562 \times 10^{10}$ \\
\hline & Pipes & - & $5.318 \times 10^{9}$ & $1.075 \times 10^{10}$ \\
\hline \multirow{3}{*}{ Investment } & Generation & $7.953 \times 10^{11}$ & $7.815 \times 10^{11}$ & $7.834 \times 10^{11}$ \\
\hline & Lines & $4.536 \times 10^{10}$ & $4.677 \times 10^{10}$ & $1.994 \times 10^{10}$ \\
\hline & Pipes & - & $4.382 \times 10^{9}$ & $1.341 \times 10^{10}$ \\
\hline \multirow{2}{*}{ Other } & Emissions & $1.438 \times 10^{11}$ & $1.458 \times 10^{11}$ & $1.745 \times 10^{11}$ \\
\hline & End Horizon & $-4.56 \times 10^{11}$ & $-4.52 \times 10^{11}$ & $-4.58 \times 10^{11}$ \\
\hline \multicolumn{2}{|c|}{ Total Cost } & $1.535 \times 10^{12}$ & $1.521 \times 10^{12}$ & $1.568 \times 10^{12}$ \\
\hline
\end{tabular}

Table 6. Final capacity comparison.

\begin{tabular}{lcccc}
\hline \multicolumn{1}{c}{ Capacity } & Unit & Model 1 & Model 2 & Model 3 \\
\hline Lines & GW & 272 & 272 & 266 \\
Pipes & MMcf/h & - & 4456 & 4871 \\
Generation & GW & 1055 & 1049 & 1014 \\
\hline Coal & GW & 9 & 9 & 11 \\
Gas & GW & 397 & 395 & 384 \\
Hydro & GW & 75 & 75 & 73 \\
Nuclear & GW & 122 & 124 & 132 \\
Offshore Wind & GW & - & - & 55 \\
Onshore Wind & GW & 442 & 436 & 347 \\
Others & GW & 11 & 11 & 12 \\
\hline
\end{tabular}

Table 7. Simulation parameters' comparison.

\begin{tabular}{lccc}
\hline \multicolumn{1}{c}{ Parameter } & Model 1 & Model 2 & Model 3 \\
\hline Simulation time (hh:mm:ss) & $1: 35: 06$ & $1: 57: 57$ & $197: 54: 10$ \\
Relative gap & 0.001757 & 0.001871 & 0.014033 \\
\hline
\end{tabular}

Finally, simulation times for Model 3 are considerably high. This is due to the computation requirements when modeling the Weymouth equations as a set of piecewise linear approximations, especially for candidate pipelines. 


\section{Conclusions}

The co-optimized analysis and design of electric and natural gas infrastructures enable identification of less costly investment alternatives, highlighting the relevance of developing new planning procedures and tools that guarantee a systematic approach for the integrated system. This issue is particularly important during a time of major expansions and even more, considering the longevity of the infrastructure to be built.

Additional modeling characteristics could be addressed to improve the problem formulation and the simulation results (e.g., losses in the transportation networks), while managing adequately the trade-off between results' accuracy and computational requirements. Sensitivity analysis of the different models could also be considered as future research in order to identify relevant model parameters.

In our work, we have developed the computational tools to facilitate gas-electric expansion planning. However, there will also need to be coordinating bodies and procedures developed because, at this point in time, the organizations that plan and build natural gas infrastructure are completely different from the organizations that plan and build electric infrastructure. Finally, because these infrastructures are interregional, decision-making processes will benefit from coordination at the national level.

Author Contributions: Conceptualization, S.L.-C. and J.M.; methodology, S.L.-C.; software, S.L.-C.; validation, S.L.-C. and J.M.; formal analysis, S.L.-C.; investigation, S.L.-C.; data curation, S.L.-C.; writing-original draft preparation, S.L.-C.; writing-review and editing, J.M.; supervision, J.M.; funding acquisition, J.M.

Funding: This work was supported in part by the Department of Energy, National Energy Technology Laboratory, under Award Number DE-OE0000316.

Conflicts of Interest: The authors declare no conflict of interest.

\section{Nomenclature}

\section{Sets (Indices)}

D compression/reduction stations $\quad(d)$

$D_{c} \quad$ compression stations $\quad(d)$

$D_{r} \quad$ reduction stations $\quad(d)$

$F$ fuels $(f)$

$G \quad$ generation units $\quad(g)$

$G_{C} \quad$ candidate generation units $(g)$

$G_{e} \quad$ existing generation units $(g)$

$I, I^{\prime}$ partitions for the squared pressure variables $\left(i, i^{\prime}\right)$

$J$ nodes $(j)$

$J_{a} \quad$ area nodes $\quad(j)$

$J_{p} \quad$ pipeline nodes $\quad(j)$

$K \quad$ generation technologies $(k)$

$K_{g} \quad$ gas-fired generation technologies $\quad(k)$

$L \quad$ transmission lines $\quad(l)$

$L_{c} \quad$ candidate transmission lines $\quad(l)$

$L_{e} \quad$ existing transmission lines $\quad(l)$

$M$ months (m)

$P$ pipelines $\quad(p)$

$P_{c} \quad$ candidate pipelines $\quad(p)$

$P_{e} \quad$ existing pipelines $\quad(p)$

$S \quad$ load blocks (s)

$T$ years $(t)$ 


\section{Symbols}

$\iota$ final year

$B_{d} \quad$ compression/reduction station start node

$B_{l} \quad$ transmission line start node

$B_{p} \quad$ pipeline start node

$E_{d}$ compression/reduction station end node

$E_{l} \quad$ transmission line end node

$E_{p} \quad$ pipeline end node

\section{Tuples (Set of Indices)}

$\bar{D}$ compression/reduction station tuple

$\bar{G}$ generation units tuple

$\bar{L} \quad$ transmission lines tuple

$\bar{d}\left(d, B_{d}, E_{d}\right)$

$\bar{P} \quad$ pipelines tuple

$\bar{g}(g, j, k)$

$\bar{l}\left(l, B_{l}, E_{l}\right)$

$\bar{p}\left(p, B_{p}, E_{p}\right)$

\section{Parameters}

$\gamma_{\bar{\delta}} \quad$ heat rate value for generator $\bar{g}$ (MMBTU/MWh)

$\eta \quad$ natural gas heat value (MMBTU/MMcf)

$\delta_{k}^{g} \quad$ generator type $k$ lifetime (years)

$\delta^{t l} \quad$ transmission line lifetime (years)

$\delta^{p l} \quad$ pipeline lifetime (years)

$\xi^{t} \quad$ discount rate in year $t(\%)$

maximum squared pressure in node $j$, year $t$, month $m$ and block $s$ (psig ${ }^{2}$ )

minimum squared pressure in node $j$, year $t$, month $m$ and block $s\left(\mathrm{psig}^{2}\right.$ )

load shedding cost (USD/MWh)

$v_{k, f} \quad$ carbon emissions for generator type $k$ and fuel $f\left(\mathrm{lb}-\mathrm{CO}_{2} / \mathrm{MMBTU}\right)$

$\Pi_{j i} \quad$ theoretical squared pressure for partition $i$ and node $j\left(\mathrm{psig}^{2}\right)$ 
$G_{j t m s}^{p, \max }$ maximum gas production rate in area $j$, year $t$, month $m$ and block $s$ (MMcf/h)

$G_{\bar{p} t m s}^{p l, m a x}$ maximum capacity for pipeline $\bar{p}$, year $t$, month $m$ and block $s$ (MMcf $/ \mathrm{h}$ )

$G_{\bar{p} t m s}^{p l, m i n}$ minimum capacity for pipeline $\bar{p}$, year $t$, month $m$ and block $s$ (MMcf $/ \mathrm{h}$ )

$h_{s} \quad$ block duration (h)

$M^{p l} \quad$ pipelines large constant

$M^{t l} \quad$ transmission lines large constant

$P_{j t m s}^{d} \quad$ power demand in area $j$, year $t$, month $m$ and block $s$ (MW)

$P_{j t}^{d, p e a k} \quad$ power peak demand in area $j$ and year $t$ (MW)

$P_{\bar{l} t m s}^{t l, m a x} \quad$ maximum capacity for transmission line $\bar{l}$ in year $t$, month $m$ and block $s$ (MW)

$P_{\bar{l} t m s}^{t l, m i n} \quad$ minimum capacity for transmission line $\bar{l}$ in year $t$, month $m$ and block $s(\mathrm{MW})$

$r_{j}$

$S^{\text {base }}$

$W_{\bar{d}} \quad$ technical coefficient for compressor/reductor $\bar{d}$

$X_{\bar{l}} \quad$ reactance for transmission line $\bar{l}$ (p.u.)

$Y_{\bar{p}} \quad$ technical coefficient for pipeline $\bar{p}\left(\mathrm{psig}^{2} /(\mathrm{MMcf} / \mathrm{h})^{2}\right)$

\section{Continuous Decision Variables}

$\Gamma_{\bar{p} t m s}^{p l} \quad$ squared gas flow across pipeline $\bar{p}$ in year $t$, month $m$ and block $s\left((\mathrm{MMcf} / \mathrm{h})^{2}\right)$

$\lambda g_{\bar{p} t m s i i^{\prime}}^{p l} \quad$ linear combination coefficients for gas flow across pipeline $\bar{p}$ in year $t$, month $m$, block $s$ and partition point $\left(i, i^{\prime}\right)$

$\lambda \pi_{j t m s i} \quad$ linear combination coefficients for squared pressure in node $j$, year $t$, month $m$, block $s$ and partition point $i$

$\pi_{j t m s} \quad$ squared pressure in node $j$, year $t$, month $m$ and block $s\left(\mathrm{psig}^{2}\right)$

$\pi_{j^{\prime} t m s} \quad$ squared pressure in node $j^{\prime}$, year $t$, month $m$ and block $s$ ( psig $\left.^{2}\right)$

$\theta_{j t m s} \quad$ voltage angle in area $j$, year $t$, month $m$ and block $s$ (rad)

$\zeta \quad$ net present cost (USD)

$C_{\bar{g} t} \quad$ number of units for generator $\bar{g}$ in year $t$ (units)

$G_{\bar{d} t m s}^{c r} \quad$ gas flow across compressor/reductor $\bar{d}$ in year $t$, month $m$ and block $s$ (MMcf/h)

$G_{\bar{d} t m s}^{\prime c r}$

$G_{j k t m s}^{P}$

$G_{j t m s}^{p, t}$

$G_{\bar{p} t m s}^{p l}$

$G_{\bar{p} t m s}^{\prime p l}$

$G_{\bar{p} t m s}$
$P_{\bar{g} t m s}^{g}$

$P_{\bar{g} t m}^{\mathcal{g}}$
$P_{j t m}^{l s}$

$P_{j t m s}^{l s}$

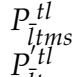
gas flow across compressor/reductor $\bar{d}$ (opposite direction) in year $t$, month $m$, and block $s$ (MMcf/h) gas production rate for area $j$, technology type $k$, year $t$, month $m$ and block $s$ (MMcf $/ \mathrm{h}$ ) total gas production rate for area $j$, year $t$, month $m$ and block $s$ (MMcf/h) gas flow across pipeline $\bar{p}$ in year $t$, month $m$ and block $s$ (MMcf/h) gas flow across pipeline $\bar{p}$ (opposite direction) in year $t$, month $m$ and block $s$ (MMcf/h) power level for generator $\bar{g}$, in year $t$, month $m$ and block $s$ (MW) load shedding in area $j$, year $t$, month $m$ and block $s$ (MW) power across transmission line $\bar{l}$ in year $t$, month $m$ and block $s$ (MW) power across transmission line $\bar{l}$ (opposite direction) in year $t$, month $m$, and block $s$ (MW)

\section{Integer Decision Variables}

$C_{\bar{g} t}^{a} \quad$ investments for generator $\bar{g}$ in year $t$ (units)

$C_{\bar{g} t}^{r} \quad$ retirements for generator $\bar{g}$ in year $t$ (units)

\section{Binary Decision Variables}

$S_{\bar{l} t} \quad$ set to 1 if transmission line $\bar{l}$ has been installed until period $t, 0$ otherwise, for $l \in L_{c}$

$S_{\bar{p} t} \quad$ set to 1 if pipeline $\bar{p}$ has been installed until period $t, 0$ otherwise, for $p \in P_{c}$

$Z_{\bar{l} t} \quad$ set to 1 if transmission line $\bar{l}$ is installed in period $t, 0$ otherwise, for $l \in L_{c}$

$Z_{\bar{p} t} \quad$ set to 1 if pipeline $\bar{p}$ is installed in period $t, 0$ otherwise, for $p \in P_{\mathcal{C}}$ 


\section{Special Ordered Sets of Type 2 (SOS2) Decision Variables}

$\lambda \pi_{j t m s i}$ linear combination coefficients for squared pressure in area $j$, year $t$, month $m$, block $s$ and partition $i$

$\lambda g_{\bar{p} t m s i}^{p l}$ linear combination coefficients for gas flow across pipe $\bar{p}$ in year $t$, month $m$, block $s$ and partition $i$

\section{References}

1. Li, T.; Eremia, M.; Shahidehpour, M. Interdependency of Natural Gas Network and Power System Security. IEEE Trans. Power Syst. 2008, 23, 1817-1824. [CrossRef]

2. North American Electric Reliability Corporation. 2011 Special Reliability Assessment: A Primer of the Natural Gas and Electric Power Interdependency in the United States; Technical Report; NERC: Atlanta, GA, USA, 2011.

3. North American Electric Reliability Corporation. 2013 Special Reliability Assessment: Accommodating an Increased Dependence on Natural Gas for Electric Power. Phase II: A Vulnerability and Scenario Assessment for the North American Bulk Power System; Technical Report; NERC: Atlanta, GA, USA, 2013.

4. Unsihuay, C.; Marangon-Lima, J.W.; de Souza, A. Integrated Power Generation and Natural Gas Expansion Planning. In Proceedings of the IEEE Lausanne Power Tech, Lausanne, Switzerland, 1-5 July 2007; pp. 1404-1409. [CrossRef]

5. Erdener, B.C.; Pambour, K.A.; Lavin, R.B.; Dengiz, B. An integrated simulation model for analysing electricity and gas systems. Int. J. Electr. Power Energy Syst. 2014, 61, 410-420. [CrossRef]

6. Clegg, S.; Mancarella, P. Integrated Electrical and Gas Network Flexibility Assessment in Low-Carbon Multi-Energy Systems. IEEE Trans. Sustain. Energy 2016, 7, 718-731. [CrossRef]

7. Clegg, S.; Mancarella, P. Integrated Modeling and Assessment of the Operational Impact of Power-to-Gas (P2G) on Electrical and Gas Transmission Networks. IEEE Trans. Sustain. Energy 2015, 6, 1234-1244. [CrossRef]

8. Qiao, Z.; Guo, Q.; Sun, H.; Pan, Z.; Liu, Y.; Xiong, W. An interval gas flow analysis in natural gas and electricity coupled networks considering the uncertainty of wind power. Appl. Energy 2017, 201, 343-353. [CrossRef]

9. Correa-Posada, C.M.; Sánchez-Martín, P. Integrated Power and Natural Gas Model for Energy Adequacy in Short-Term Operation. IEEE Trans. Power Syst. 2015, 30, 3347-3355. [CrossRef]

10. Zlotnik, A.; Roald, L.; Backhaus, S.; Chertkov, M.; Andersson, G. Coordinated Scheduling for Interdependent Electric Power and Natural Gas Infrastructures. IEEE Trans. Power Syst. 2017, 32, 600-610. [CrossRef]

11. Zheng, J.; Wu, Q.; Jing, Z. Coordinated scheduling strategy to optimize conflicting benefits for daily operation of integrated electricity and gas networks. Appl. Energy 2017, 192, 370-381. [CrossRef]

12. Quelhas, A.; Gil, E.; McCalley, J.; Ryan, S. A Multiperiod Generalized Network Flow Model of the U.S. Integrated Energy System: Part I-Model Description. IEEE Trans. Power Syst. 2007, 22, 829-836. [CrossRef]

13. Quelhas, A.; McCalley, J. A Multiperiod Generalized Network Flow Model of the U.S. Integrated Energy System: Part II—Simulation Results. IEEE Trans. Power Syst. 2007, 22, 837-844. [CrossRef]

14. Unsihuay-Vila, C.; Marangon-Lima, J.W.; de Souza, A.; Perez-Arriaga, I.; Balestrassi, P. A Model to Long-Term, Multiarea, Multistage, and Integrated Expansion Planning of Electricity and Natural Gas Systems. IEEE Trans. Power Syst. 2010, 25, 1154-1168. [CrossRef]

15. Bahiense, L.; Oliveira, G.C.; Pereira, M.; Granville, S. A Mixed Integer Disjunctive Model for Transmission Network Expansion. IEEE Trans. Power Syst. 2001, 16, 560-565. [CrossRef]

16. Barati, F.; Nateghi, A.; Seifi, H.; Sepasian, M. Generation and transmission expansion planning with considering natural gas network. In Proceedings of the 21st Iranian Conference on Electrical Engineering (ICEE), Mashhad, Iran, 14-16 May 2013, pp. 1-7. [CrossRef]

17. Behrouzpanah, M.; Sepasian, M.; Bayat, S. Subtransmission system expansion planning with dgs considering natural gas transmission constraints. In Proceedings of the 4th Conference on Thermal Power Plants, Tehran, Iran, 18-19 December 2012; pp. 1-6.

18. Chaudry, M.; Jenkins, N.; Qadrdan, M.; Wu, J. Combined gas and electricity network expansion planning. Appl. Energy 2014, 113, 1171-1187. [CrossRef]

19. Hu, Y.; Bie, Z.; Ding, T.; Lin, Y. An NSGA-II based multi-objective optimization for combined gas and electricity network expansion planning. Appl. Energy 2016, 167, 280-293. [CrossRef] 
20. Khaligh, V.; Oloomi Buygi, M.; Anvari-Moghaddam, A.; M. Guerrero, J. A Multi-Attribute Expansion Planning Model for Integrated Gas-Electricity System. Energies 2018, 11, 2573. [CrossRef]

21. Liu, A.; Hobbs, B.; Ho, J.; McCalley, J.; Krishnan, V.; Shahidehpour, M.; Zheng, Q. Co-Optimization of Transmission and Other Supply Resources; White Paper; NARUC: Washington, DC, USA, 2013.

22. Romero, R.; Monticelli, A.; Garcia, A.; Haffner, S. Test systems and mathematical models for transmission network expansion planning. IEE Proc. Gener. Transm. Distrib. 2002, 149, 27-36.:20020026. [CrossRef]

23. Binato, S. Optimal Expansion of Transmission Networks by Benders Decomposition and Cutting Planes. Ph.D. Thesis, University of Rio de Janeiro, Rio de Janeiro, Brazil, 2000.

24. Li, Y. Transmission Design and Optimization at the National Level. Ph.D. Thesis, Iowa State University, Ames, IA, USA, 2014.

25. Martin, A.; Möller, M.; Moritz, S. Mixed Integer Models for the Stationary Case of Gas Network Optimization. Math. Programm. 2006, 105, 563-582. [CrossRef]

26. Urbina, M.; Li, Z. A Combined Model for Analyzing the Interdependency of Electrical and Gas Systems. In Proceedings of the 39th North American Power Symposium, as Cruces, NM, USA, 30 September-2 October 2007; pp. 468-472. [CrossRef]

27. The Eastern Interconnection Planning Collaborative Website. Phase II Documents. DOE Award Project DE-OE0000343, 2015. Available online: https://www.eipconline.com/phase-ii-documents (accessed on 20 March 2019).

28. Lemos-Cano, S. Co-Optimized Analysis of Electric and Natural Gas Infrastructures. Ph.D. Thesis, Iowa State University, Ames, IA, USA, 2017.

(C) 2019 by the authors. Licensee MDPI, Basel, Switzerland. This article is an open access article distributed under the terms and conditions of the Creative Commons Attribution (CC BY) license (http:/ / creativecommons.org/licenses/by/4.0/). 